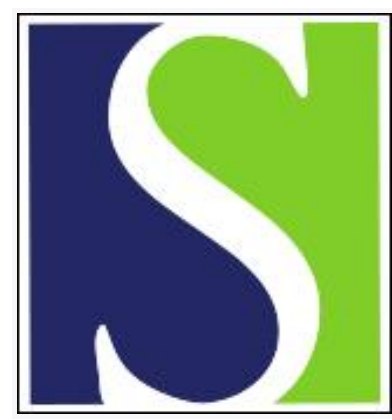

Scand J Work Environ Health 2017;43(1):96

https://doi.org/10.5271/sjweh.3609

Published online: 09 Dec 2016, Issue date: 01 Jan 2017

Response to Dr Stevens' letter ref. Visitisen et al: “Short-term effects of night shift work on breast cancer risk: a cohort study of payroll data"

by Kolstad HA, Garde AH, Hansen ÅM, Frydenberg M, Christiansen P, Vistisen $\mathrm{HT}$, Bonde JPE

Affiliation: Department of Occupational Medicine, Danish Ramazzini Centre, Aarhus University Hospital, Aarhus, Denmark. henkol@rm.dk

Refers to the following texts of the Journal: 2013;39(5):431-447 2017;43(1):59-67 2017;43(1):95

Key terms: breast cancer; cancer; circadian disruption; cohort study; effect; epidemiology; letter; night shift work; night work; payroll data; shift work; shift worker; working time

This article in PubMed: www.ncbi.nlm.nih.gov/pubmed/27935622 


\section{Response to Dr Stevens' letter ref. Visitisen et al: "Short-term effects of night shift work on breast cancer risk: a cohort study of payroll data"}

We thank Dr Richard Stevens for his comments (1) on our recent article that showed no increased risk of breast cancer following recent night shift work when compared with recent day shift work (2). This finding was based on linkage of day-by-day information on working hours and breast cancer incidence data. Results are thus less likely to have been biased by differential misclassification than findings from earlier studies relying on self-report (3).

We defined a night shift as $\geq 3$ hours of work between 24:00-05:00 hours and a day shift as $\geq 3$ hours work between 6:00-20:00 hours. This day shift definition did not exclude shifts starting before 05:00 or ending after 24:00 hours. However, this affected only $0.02 \%$ and $0.3 \%$ of all day shifts, respectively. This diminutive misclassification, that is expected to be non-differential, can hardly explain our negative findings.

It is suggested that shifts that begin after 07:00 and end before 18:00 would constitute a more sensible baseline comparison group. Since the biological mechanism is not certain, it is not obvious to us if this will be a more appropriate reference than the present. However, we agree that future studies should test how different definitions of shifts affect the risk of breast cancer, which will be possible using this type of data.

We only had information on working hours from 2007 and onwards, and night shift work prior to 2007 could have confounded our analyses towards no effect but only if inversely associated with night shift work in 2007 or later. We find this unlikely. Left truncation could also have biased findings towards the null. We therefore supplemented analyses of the total study population with analyses of the one-third of the population with first recorded employment in 2008 or later (the inception population). Even if the mean age was 35.5 years - and many undoubtedly had been working (with and without night shifts) prior to 2008 - this population should be less affected by such selection bias, but we observed similar risk estimates as for the total study population.

Taken together, we find that our study provides rather robust evidence of no short-term breast cancer risk following recent night shift work. It must, however, be stressed that data did not allow assessment of a possible long-term risk.

\section{Reference}

1. Stevens R. Letter ref. Vitisen et al: "Short-term effects of night shift work on breast cancer risk: a cohort study of payroll data". Scand J Work Environ Health. 2017;43(1):95. http://dx.doi.org/10.5271/ sjweh.3607

2. Vistisen HT, Garde AH, Frydenberg M, Christiansen P, Hansen AM, Hansen J, Bonde JP, Kolstad HA. Short-term effects of night shift work on breast cancer risk: A cohort study of payroll data. Scand J Work Environ Health. 2017;43(1):59-67. http:// dx.doi.org/10.5271/sjweh.3603.

3. Ijaz S, Verbeek J, Seidler A, Lindbohm ML, Ojajarvi A, Orsini N, Costa G, Neuvonen K. Night-shift work and breast cancer--a systematic review and meta-analysis. Scand J Work Environ Health. 2013 Sep 1;39(5):431-47. http://dx.doi.org/10.5271/ sjweh.3371

Henrik A Kolstad, MD, ${ }^{1}$ Anne Helene Garde, PhD, ${ }^{2}$ Åse Marie Hansen, PhD, ${ }^{2,3}$ Morten Frydenberg, PhD, ${ }^{4}$ Peer Christiansen, $M D,{ }^{5,6}$ Helene Tilma Vistisen, PhD, ${ }^{1}$ Jens Peter E Bonde, MD, ${ }^{7}$

1 Department of Occupational Medicine, Danish Ramazzini Centre, Aarhus University Hospital, Aarhus, Denmark.

2 National Research Centre for the Working Environment, Copenhagen, Denmark.

3 Department of Public Health, University of Copenhagen, Copenhagen, Denmark.

4 Section for Biostatistics, Department of Public Health, Aarhus University, Aarhus, Denmark.

5 Department of Breast Surgery, Aarhus University Hospital, Aarhus, Denmark.

6 Danish Breast Cancer Corporative Group (DBCG). http://www. dbcg.dk/

7 Department of Occupational and Environmental Medicine, Bispebjerg University Hospital, Copenhagen, Denmark.

Correspondence to: Henrik A Kolstad, Department of Occupational Medicine, Danish Ramazzini Centre, Aarhus University Hospital, Aarhus, Denmark. [E-mail:henkol@rm.dk]. 